\title{
PSOROSA MEDITERRANELLA (AMSEL, 1954) (LEPIDOPTERA: PYRALIDAE, PHYCITINAE) - A NEW SPECIES FOR THE CROATIAN PYRALOID MOTH FAUNA, WITH AN UPDATED CHECKLIST
}

\section{Danijela Gumhalter}

Azuritweg 2, 70619 Stuttgart, Germany (e-mail: danijela.gumhalter@gmail.com)

Gumhalter, D.: Psorosa mediterranella (Amsel, 1954) (Lepidoptera: Pyralidae, Phycitinae) - a new species for the Croatian pyraloid moth fauna, with an updated checklist. Nat. Croat., Vol. 30, No. 1, 37-52, 2021, Zagreb.

From 2016 to 2020 numerous surveys were undertaken to improve the knowledge of the pyraloid moth fauna of Biokovo Nature Park. On August 27 th 2020 one specimen of Psorosa mediterranella (Amsel, 1954) from the family Pyralidae was collected on a small meadow (985 m a.s.l.) on Mt Biokovo.

In this paper, the first data about the occurrence of this species in Croatia are presented. The previous mention in the literature for Croatia was considered to be a misidentification of the past and has thus not been included in the checklist of Croatian pyraloid moth species.

P. mediterranella was recorded for the first time in Croatia in recent investigations and, after other additions to the checklist have been counted, is the $396^{\text {th }}$ species in the Croatian pyraloid moth fauna. An overview of the overall pyraloid moth fauna of Croatia is given in the updated species list.

Keywords: Psorosa mediterranella, Pyraloidea, Pyralidae, fauna, Biokovo, Croatia

Gumhalter, D.: Psorosa mediterranella (Amsel, 1954) (Lepidoptera: Pyralidae, Phycitinae) nova vrsta u hrvatskoj fauni Pyraloidea, s nadopunjenim popisom vrsta. Nat. Croat., Vol. 30, No. 1, 37-52, 2021, Zagreb.

Kako bi se doprinijelo poznavanju faune Pyraloidea Parka prirode Biokovo, od 2016. do 2020. godine proveden je velik broj faunističkih istraživanja. Tijekom terenskog istraživanja 27. kolovoza 2020. godine ulovljen je jedan primjerak vrste Psorosa mediterranella (Amsel, 1954) iz porodice Pyralidae. Primjerak je skupljen na maloj livadi na planini Biokovo ( $985 \mathrm{~m}$ n.v.).

U radu se navodi prvi nalaz ove vrste za faunu Pyraloidea Hrvatske. Iako se vrsta prethodno spominjala u literaturi za Hrvatsku, polazilo se od pretpostavke da se radi o pogrešnoj determinacije te $P$. mediterranella nije bila uvrštena u popis vrsta hrvatskih Pyraloidea.

Vrsta $P$. mediterranella je zabilježena prvi puta u recentnim istraživanjima te je, zajedno s drugim dodacima popisu vrsta, 396. vrsta u hrvatskoj fauni Pyraloidea.

Ključne riječi: Psorosa mediterranella, Pyraloidea, Pyralidae, fauna, Biokovo, Hrvatska

\section{INTRODUCTION}

The 2019 checklist of Pyraloidea species in Croatia included 377 species (GumHaLTER, 2019a; 2019b). This checklist was a result of a detailed faunistic analysis of the Pyraloidea fauna in Croatia, which included extensive literature research, examinations of museum collections and results from own field investigations. From 2015 until 2020, the author conducted numerous surveys at different localities in Croatia to 
contribute to the knowledge of the overall pyraloid moth fauna of the country. More than a third of all species from the checklist were recorded throughout these field surveys. All the material collected during this extensive study was deposited in the private collection of the author (coll. Gumhalter).

Subsequently published data about additional new species from the families Pyralidae and Crambidae in Croatia have shown that the Croatian fauna of Pyraloidea is not yet thoroughly known. In 2019, SLAMKa published the fourth volume of the "Pyraloidea of Europe", covering six more species that have not been previously listed in the species list of the Croatian Pyraloidea. More additions have to be made, as two species were previously overlooked in the literature (REBEL, 1901; SLAMKA, 2008). After the publication of the checklist Koren (2020) wrote an article on three new montane species from Croatia, but only two of these will be added to the checklist, as one species was already mentioned in the literature. Another article concerning one new species was published the same year (Koren \& Kuljer, 2020) and the following year as well (KOREN, 2021a).

These new findings were added to the species list and an updated checklist is presented here. Although further additions to the checklist are to be expected, Croatia can already be seen to have a very diverse Pyraloidea fauna compared to neighboring countries (Gumhalter, 2020).

Since little is known about the occurrence of Pyraloidea species from the Croatian mountains, there is a great need to explore these areas in the future. In numerous field investigations conducted from 2016 to 2020 on Mt Biokovo, a total of 77 Pyraloidea species were reported (Gumhalter \& Kučinić, 2021). During the last moth survey, one specimen of Psorosa mediterranella (Amsel, 1954) from the family Pyralidae was collected (Fig. 1). In the recent investigations the species P. mediterranella has been recorded for the first time in Croatia. The specimen was collected at an altitude of approximately 985 meters above sea level $\left(43^{\circ} 26^{\prime} 68.0^{\prime \prime} \mathrm{N} 17^{\circ} 10^{\prime} 27.7^{\prime \prime} \mathrm{E}\right)$.

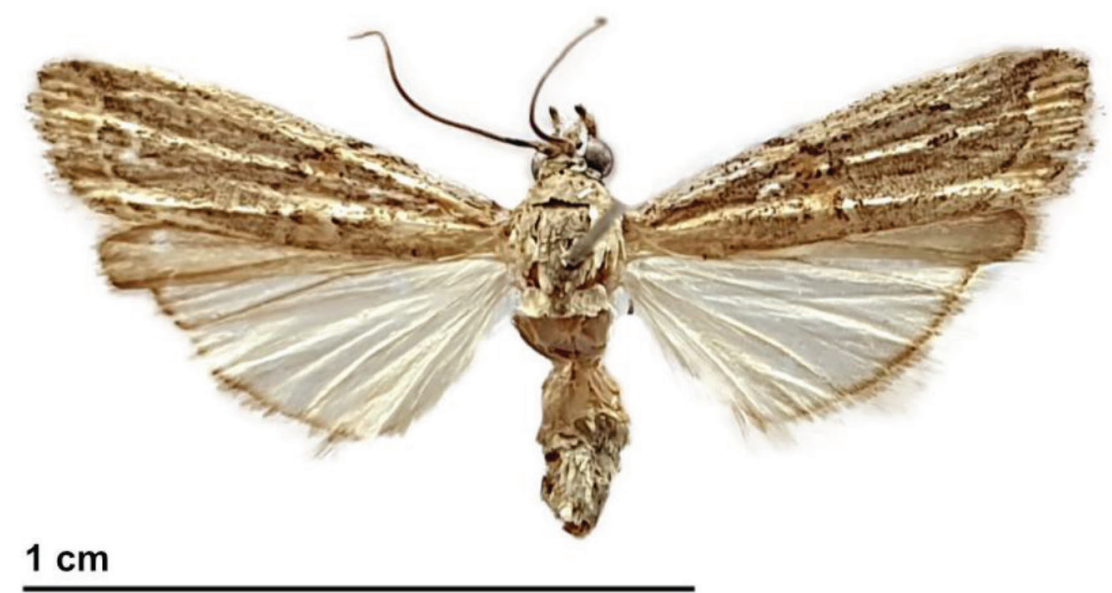

Fig. 1. A female specimen of P. mediterranella (wingspan $16 \mathrm{~mm}$ ) collected on $27^{\text {th }}$ August 2020 on Mount Biokovo (photo: D. Gumhalter). 
Including the above-mentioned additions, the number of Pyraloidea species occurring in Croatia has risen to 396.

The aim of this study is to present the occurrence of the new species $P$. mediterranella in the fauna of Croatia. This paper also provides an updated list of all pyraloid moth species recorded in Croatia (Appendix 1).

\section{MATERIALS AND METHODS}

The author carried out research on Pyraloidea fauna from Biokovo Nature Park from 2016 to 2020 (Fig. 2). All specimens were collected at several localities on the southern slopes of Mount Biokovo at elevations ranging from 270 to 1762 meters above sea level. The specimens were caught with a UV light and deposited in the private collection of the author (coll. Gumhalter). Amongst these findings was the collected specimen of $P$. mediterranella, which was also deposited in the Gumhalter Collection. The determination of the species was conducted according to SLAmkA (2019).

\section{RESULTS AND DISCUSSION}

As stated by van Nieukerken et al. (2011) the family Pyralidae currently includes 5,921 described species worldwide. The genus Psorosa Zeller, 1846 is a rather small genus with only six species present in the European pyralid moth fauna (Nuss et al., 2013).

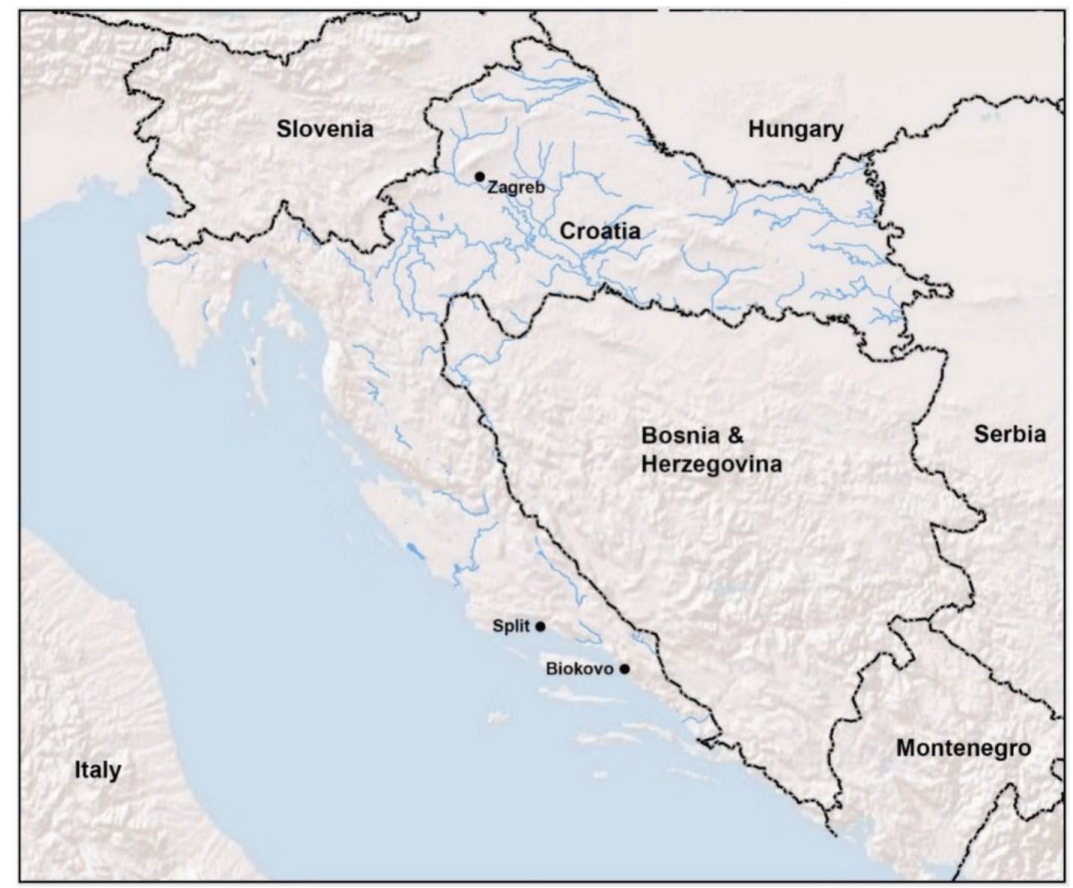

Fig. 2. Map with the position of Mount Biokovo in Croatia. 
The male holotype of $P$. mediterranella was described from Spain (Amsel, 1954) and originates from the year 1926. In 1930, Amsel collected one female allotype from Dubrovnik in Dalmatia. As reported by Nuss et al. (2013) the species is also listed in the Fauna Europaea database as present in the fauna of Croatia. However, the only source Nuss et al. provide is the work of Klimesch (1942), who reported only P. nucleolella (Möschler, 1866) from Croatia. According to Fauna Europaea, however, P. nucleolella is not present in the fauna of Croatia. Although the species has been mentioned in the literature for Croatia, it was unclear if the determination of $P$. mediterranella could be considered a misidentification. Therefore, $P$. mediterranella was previously not included in the checklist of Croatian pyraloid moth species.

According to Plant (2016), the species P. mediterranella and P. nucleolella (Möschler, 1866) have been confused in eastern and western Europe. Correct identification is only possible by examination of the genitalia (LERAUT, 2007), as the adults of both species are identical in appearance. Although $P$. nucleolella has been previously mentioned in the literature for Croatia (KLIMesch, 1942) it is unclear whether it was really $P$. nucleolella or whether it had been confused with $P$. mediterranella. The same applies to Amsel's identification of $P$. mediterranella. Since no genitalia analysis was done, it is probable that the specimen in question was in fact $P$. nucleolella. Following PLANT (2016), it seems clear that P. nucleolella is the dominant species in the east. However, the eastern limit of the range of $P$. mediterranella is unknown and it is possible that the same two species may have been confused in eastern Europe as well as in the west. Plant concludes that it is likely that $P$. mediterranella is a species that has been overlooked throughout the Balkan Peninsula.

Without strong evidence of its occurrence in Croatia, P. mediterranella was previously excluded from the checklist.

In 2020 P. mediterranella was found in Dalmatia on Mt Biokovo, on a small rocky meadow along the road which leads from the entrance of the Nature Park right to the peak of Sveti Jure (Fig. 3). One pale male specimen was collected at an altitude of approximately 985 meters above sea level. To assure identification, genitalia slides were made. The analysis showed clearly that the collected specimen is P. mediterranella.

According to Slamka (2019), P. mediterranella inhabits arid open habitats, steppes up to 2,200 $\mathrm{m}$ a.s.l. and has a wingspan from 16 up to $22 \mathrm{~mm}$. The species is distributed in Portugal, Spain and the Canary Islands, France, Corsica, Italy (Sicily), Balkan countries, and outside of Europe in Morocco, Algeria and Tunisia.

The unclear status about the occurrence of this species in Croatia has thus been resolved and P. mediterranella can be added to the Croatian fauna of Pyraloidea species.

Also, the following six species were extracted from the fourth volume of the "Pyraloidea of Europe" (SLAmKa, 2019) and were added to the species list: Cryptoblabes gnidiella (Millière, 1867), Elegia atrifasciella (Ragonot, 1887), Merulempista amoenella (Zeller, 1848), M. brucella (Staudinger, 1879), Pempelia compositella (Treitschke, 1835) and Phycita acericola (Kuznetzov, 1960).

Besides, Slamka (2019) reports the species Jerichoa mediterranella (Amsel, 1935) for Croatia by citing Amsel's work. According to Amsel, the species is distributed in Palestine and Sicily. Amsel also mentioned one specimen from Dalmatia, but without giving more data that are accurate about the locality. As this finding is not a confirmed one, only a historical record, J. mediterranella was excluded from the updated species list. 


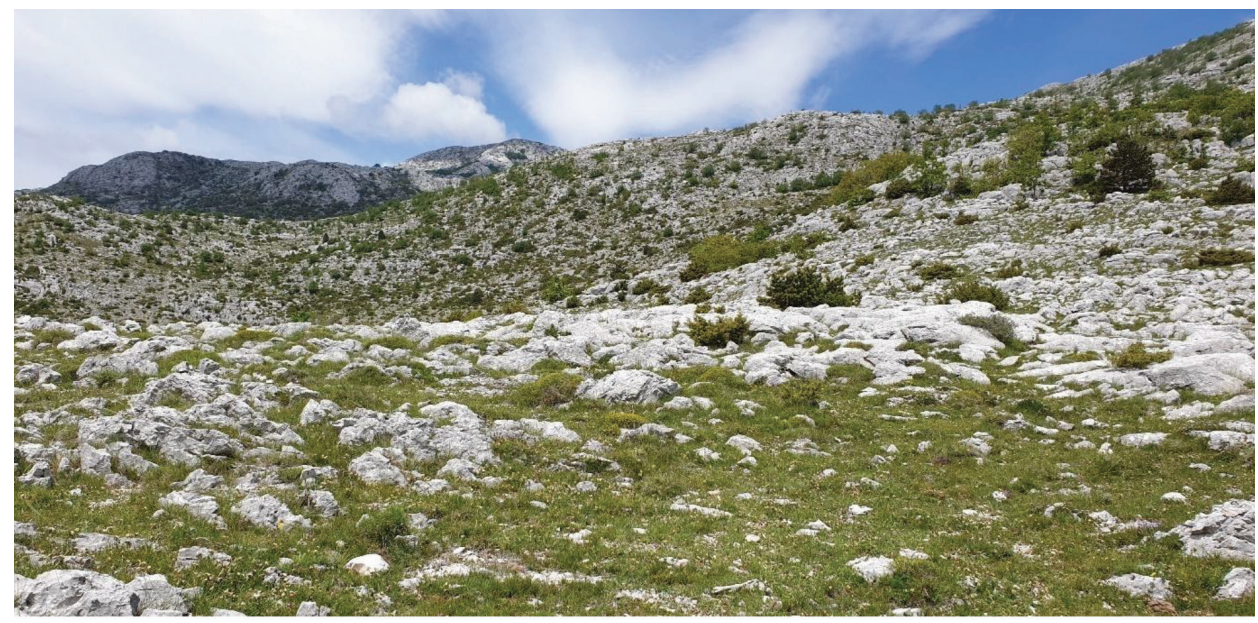

Fig. 3. A small meadow along the road where the specimen of P. mediterranella was collected in 2020 (photo: D. Gumhalter).

Likewise added to the checklist are the species Evergestis isatidalis (Duponchel, 1833) and Catoptria myella (Hübner, 1796), which were previously overlooked in the literature (Rebel, 1901; Slamka, 2008). Fauna Europaea lists both species as present in the fauna of Croatia.

After the publication of the first checklist Koren (2020) wrote an article on three new montane species from Croatia: Catoptria pyramidellus (Treitschke, 1832), Crambus ericella (Hübner, 1813) and Udea alpinalis (Denis \& Schiffermüller, 1775), all from the family Crambidae. For the reason that the species C. pyramidellus was previously mentioned by Plant \& JAKŠIć (2018) and was already listed under the number 38 in the revised checklist of Pyraloidea species in Croatia (Gumhalter, 2019b), only C. ericella and $U$. alpinalis can be added to the checklist. The finding of Friedlanderia cicatricella (Hübner, 1824) was published shortly afterward (KOREN \& Kuljer, 2020), as as was the finding of Dioryctria robiniella (Millière, 1865) in 2021 (KoREN, 2021a).

One more overlooked species from the literature has to be mentioned here. There is one historical record of Pionea thalalis (Zerny, 1914) for Croatia. The source Zerny gives is the work of MANn (1869), who reported the species under the name Botys numeralis from Croatia. According to GlobIZ, $P$. thalalis is synonymized with Eudorea simplicella. The species E. simplicella was first described by La Harpe in 1861 and is, according to Fauna Europaea a synonym of Udea numeralis. Following the database, $U$. numeralis is known from Canary Islands, Corsica, the French mainland, Gibraltar, the Greek mainland, the Italian mainland, Kriti (Crete), Madeira Island, the Portuguese mainland, Sardinia, the Spanish mainland. The species is as well present in the fauna of Croatia. Although SLAmka (2013) states that U. simplicella was previously synonymized with $U$. numeralis, he lists both $U$. simplicella and $U$. numeralis as separate species in his book, because the male genitalia differentiate one from another. Besides, $U$. simplicella is apparently smaller than $U$. numeralis and therefore treated as a different species (František Slamka, pers. comm., 09.02.2021). As reported by SLAMKA (2013), U. simplicella is distributed in southern Italy, Sicily, Tunis and Morocco. Sometime in the future, DNA analysis of four collected specimens of $U$. simplicella from 
Spain will be conducted (John Girdley, pers. comm. 10.02.2021). The results will show if $U$. simplicella and $U$. numeralis are indeed different species. As this is still unclear, and there is only one sole historical record of $U$. simplicella for Croatia, the species was excluded from the checklist. Further studies on the potential occurrence of $U$. simplicella in Croatia are recommended.

Taking into account six more species published in another article in this issue (Koren, 2021b), and by adding P. mediterranella to the checklist, the number of Pyraloidea species occurring in Croatia rises to 396 and includes 218 Crambidae and 178 Pyralidae taxa. An overview of the overall pyraloid moth fauna of Croatia is given in the updated species list in Appendix 1.

\section{ACKNOWLEDGEMENTS}

The author is grateful to Martina Šašić from the Croatian Natural History Museum in Zagreb, as well as to František Slamka and John Girdley for providing useful information on the species $U$. simplicella.

Received February 12, 2020

\section{REFERENCES}

Amsel, H. G., 1935: Neue palästinensische Lepidopteren. Mitteilungen aus dem Zoologischen Museum in Berlin 20(2), 271-319, pl. 9-18.

Amsel, H. G., 1954: Die Microlepidopteren der Brandt'schen Iran-Ausbeute. 4. Teil. Arkiv für Zoologie 6(16), 255-326, pl. I-XXXIII.

Gumhalter, D., 2019a: First checklist of pyraloid moths (Lepidoptera: Pyraloidea) in Croatia. Zootaxa 4604(1), 059-102. https:/ / doi.org/10.11646/zootaxa.4604.1.3

Gumhalter, D., 2019b: A revised checklist of pyraloid moths (Lepidoptera: Pyraloidea) in Croatia. Natura Croatica 28(2), 271-288. DOI: 10.20302/NC.2019.28.20

Gumhalter, D., 2020: Biodiversity, ecological and biogeographical features of Pyralidae and Crambidae (Insecta, Lepidoptera) in three Croatian climate regions. PhD Dissertation, University of Zagreb, Zagreb, 369 pp. (in Croatian)

Gumhalter, D. \& Kučinić, M., 2021: Contribution to the knowledge of the Croatian Pyraloidea fauna. Species reported from Biokovo Natural Park (Insecta: Lepidoptera). SHILAP Revista de lepidopterología, 49(193), 65-83.

Klimesch, J., 1942: Über Microlepidopteren-Ausbeuten von Zaton bei Gravosa (Süddalmatien) in Mitteilungen Münchener entomologischen Gesellschaft 32, 347-399, pl. 13-15.

Koren, T., 2021a: Three montane grass moths (Lepidoptera: Crambidae) new to the fauna of Croatia. Acta Entomologica Serbica 25(1), 29-34. DOI: 10.5281 /zenodo.3735368

Koren, T., 2021b: Further additions to the grass moth (Lepidoptera: Crambidae) fauna of Croatia. Natura Croatica 30(1), 243-250.

Koren, T., 2021: Dioryctria robiniella (Millière, 1865) (Lepidoptera: Pyralidae) is a member of fauna of Croatia. Acta Entomologica Serbica 26 (1), X-XX. DOI: 10.5281/zenodo.4551159

Koren, T. \& Kuljuer, D., 2020: Additions to the Crambidae (Insecta: Lepidoptera) fauna of Croatia and Bosnia \& Herzegovina. Acta Entomologica Slovenica 28(2), 141-148.

La Harpe, J.,1860: Contributions à la faune de la Sicile. Lépidoptères. Bulletin de la Société vaudoise des sciences naturelles 6(47), 386-418.

Leraut, P. J. A., 2007: Ebauche d'une liste des pyrales de France [Lepidoptera, Pyraloidea]. Revue française d'Entomologie (N.S.) 29, 149-166.

MANN, J. J., 1869: Lepidopteren, gesammelt während dreier Reisen nach Dalmatien in den Jahren 1850, 1862, 1868. Verhandlungen des Zoologisch-Botanischen Gesellschaft in Wien 19, 371-388.

Nieukerken van, E. J., Kaila, L. \& Kitching, I. J., 2011: Order Lepidoptera. Animal biodiversity: An outline of higher-level classification and survey of taxonomic richness. Zootaxa 3148, 212-221. 
Nuss, M., Landry, B., Mally, R., Vegliante, F., Tränkner, A., Bauer, F., Hayden, J., Segerer, A., Schouten, R., Li, H., Trofimova, T., Solis, M. A., De Prins, J. \& Speidel, W., 2003-2021: Global Information System on Pyraloidea, www.pyraloidea.org (Accessed 24 January 2021)

Nuss, M., Speidel, W. \& Segerer, A., 2013: Fauna Europaea: Pyralidae. In: Karsholt, O. \& Nieukerken, E. J. van: Fauna Europaea: Lepidoptera, Moths. Fauna Europaea version 2017.06, https://fauna-eu.org (Accessed 02 February 2021)

Plant, C. W., 2016: An Annotated Systematic, Synonymic and Distributional Checklist of the Pyraloidea of Bulgaria. Neue Entomologische Nachrichten 72, 1-231.

Plant, C. W. \& Jakšıć, P., 2018: A provisional checklist and bibliography of the Pyraloidea of the Balkan Peninsula (Lepidoptera: Pyralidae \& Crambidae). Atalanta 49, 219-263.

Rebel, H., 1901: II. Theil Famil. Pyralidae-Micropterygidae. In: Staudinger, O. \& Rebel, H., 1901: Catalog der Lepidopteren des Palaearctischen Faunengebietes. Berlin, 368 pp.

Slamka, F., 2006: Pyralinae, Galleriinae, Epipaschiinae, Cathariinae \& Odontiinae. Pyraloidea of Europe, Bratislava 1, 1-138.

SLAmka, F., 2008: Pyraloidea of Europe (Lepidoptera). Volumen 2. Crambinae \& Schoenobiinae. František Slamka, Bratislava, $224 \mathrm{pp}$.

SLAmKa, F., 2013: Pyraloidea of Europe (Lepidoptera). Volume 3. Pyraustinae \& Spilomelinae. Identification - Distribution - Habitat - Biology. František Slamka, Bratislava, 357 pp.

Slamka, F., 2019: Pyraloidea of Europe (Lepidoptera). Phycitinae - Volumen 4, Part 1. Identification Distribution - Habitat - Biologie 4, František Slamka, Bratislava, 432 pp.

Zerny, H., 1914: Über paläarktische Pyraliden des k. k. naturhistorischen Hofmuseums in Wien Annalen des Naturhistorischen Museums in Wien 28, 295-348.

\section{APPENDIX 1.}

An updated checklist of Pyraloidea species (Crambidae and Pyralidae) occurring in Croatia. The list of species in each genus is organized alphabetically and the systematic presentation follows the Fauna Europaea database (Nuss et al., 2013).

\section{Family Crambidae}

1. Cataclysta lemnata (Linnaeus, 1758)

2. Elophila nymphaeata (Linnaeus, 1758)

3. Elophila rivulalis (Duponchel, 1834)

4. Nymphula nitidulata (Hufnagel, 1767)

5. Parapoynx nivalis (Denis \& Schiffermüller, 1775)

6. Parapoynx stratiotata (Linnaeus, 1758)

7. Agriphila brioniellus (Zerny, 1914)

8. Agriphila dalmatinellus (Hampson, 1900)

9. Agriphila deliella (Hübner, 1813)

10. Agriphila geniculea (Haworth, 1811)

11. Agriphila inquinatella (Denis \& Schiffermüller, 1775)

12. Agriphila latistria (Haworth, 1811)

13. Agriphila paleatellus (Zeller, 1847)

14. Agriphila poliellus (Treitschke, 1832)

15. Agriphila selasella (Hübner, 1813)

16. Agriphila straminella (Denis \& Schiffermüller, 1775)

17. Agriphila tersellus (Lederer, 1855)

18. Agriphila tolli (Bleszyński, 1952)

19. Agriphila tristella (Denis \& Schiffermüller, 1775) 
20. Ancylolomia palpella (Denis \& Schiffermüller, 1775)

21. Ancylolomia pectinatellus (Zeller, 1847)

22. Ancylolomia tentaculella (Hübner, 1796)

23. Angustalius malacellus (Duponchel, 1836)

24. Calamotropha aureliellus (Fischer v. Röslerstamm, 1841)

25. Calamotropha paludella (Hübner, 1824)

26. Catoptria acutangulellus (Herrich-Schäffer, 1847)

27. Catoptria combinella (Denis \& Schiffermüller, 1775)

28. Catoptria falsella (Denis \& Schiffermüller, 1775)

29. Catoptria fulgidella (Hübner, 1813)

30. Catoptria languidellus (Zeller, 1863)

31. Catoptria luctiferella (Hübner, 1813)

32. Catoptria lythargyrella (Hübner, 1796)

33. Catoptria margaritella (Denis \& Schiffermüller, 1775)

34. Catoptria myella (Hübner, 1796)

35. Catoptria mytilella (Hübner, 1805)

36. Catoptria osthelderi (Lattin, 1950)

37. Catoptria pauperellus (Treitschke, 1832)

38. Catoptria petrificella (Hübner, 1796)

39. Catoptria pinella (Linnaeus, 1758)

40. Catoptria pyramidellus (Treitschke, 1832)

41. Catoptria verellus (Zincken, 1817)

42. Chilo luteellus (Motschulsky, 1866)

43. Chilo phragmitella (Hübner, 1805)

44. Chilo pulverosellus (Ragonot, 1895)

45. Chrysocrambus cassentiniellus (Herrich-Schäffer, 1848)

46. Chrysocrambus craterellus (Scopoli, 1763)

47. Chrysocrambus linetella (Fabricius, 1781)

48. Chrysoteuchia culmella (Linnaeus, 1758)

49. Crambus ericella (Hübner, 1813)

50. Crambus lathoniellus (Zincken, 1817)

51. Crambus pascuella (Linnaeus, 1758)

52. Crambus perlella (Scopoli, 1763)

53. Crambus pratella (Linnaeus, 1758)

54. Crambus silvella (Hübner, 1813)

55. Crambus uliginosellus (Zeller, 1850)

56. Euchromius bella (Hübner, 1796)

57. Euchromius cambridgei (Zeller, 1867)

58. Euchromius ocellea (Haworth, 1811)

59. Euchromius ramburiellus (Duponchel, 1836)

60. Euchromius rayatellus (Amsel, 1949)

61. Euchromius superbellus (Zeller, 1849) 
62. Friedlanderia cicatricella (Hübner, 1824)

63. Mesocrambus candiellus (Herrich-Schäffer, 1848)

64. Metacrambus carectellus (Zeller, 1847)

65. Pediasia aridella (Thunberg, 1788)

66. Pediasia contaminella (Hübner, 1796)

67. Pediasia fascelinella (Hübner, 1813)

68. Pediasia jucundellus (Herrich-Schäffer, 1847)

69. Pediasia luteella (Denis \& Schiffermüller, 1775)

70. [Pediasia pedriolellus (Duponchel, 1836)]

71. Pediasia siculellus (Duponchel, 1836)

72. Platytes alpinella (Hübner, 1813)

73. Platytes cerussella (Denis \& Schiffermüller, 1775)

74. Talis quercella (Denis \& Schiffermüller, 1775)

75. Thisanotia chrysonuchella (Scopoli, 1763)

76. Thopeutis galleriellus (Ragnot in Staudiger, 1892)

77. Xanthocrambus lucellus (Herrich-Schäffer, 1848)

78. Xanthocrambus saxonellus (Zincken, 1821)

79. Cybalomia lutosalis (Mann, 1862)

80. Hyperlais argillacealis (Zeller, 1847)

81. Hyperlais dulcinalis (Treitschke, 1835)

82. Hyperlais nemausalis (Duponchel, 1834)

83. Evergestis aenealis (Denis \& Schiffermüller, 1775)

84. Evergestis caesialis (Herrich-Schäffer, 1849)

85. Evergestis extimalis (Scopoli, 1763)

86. Evergestis forficalis (Linnaeus, 1758)

87. Evergestis frumentalis (Linnaeus, 1761)

88. Evergestis isatidalis (Duponchel, 1833)

89. Evergestis limbata (Linnaeus, 1767)

90. Evergestis pallidata (Hufnagel, 1767)

91. Evergestis politalis (Denis \& Schiffermüller, 1775)

92. Evergestis sophialis (Fabricius, 1787)

93. Orenaia preisseckeri (Rebel 1903)

94. Hellula undalis (Fabricius, 1775)

95. Hydriris ornatalis (Duponchel, 1832)

96. Eurrhypis guttulalis (Herrich-Schäffer, 1848)

97. Eurrhypis pollinalis (Denis \& Schiffermüller, 1775)

98. Aporodes floralis (Hübner, 1809)

99. Atralata albofascialis (Treitschke, 1829)

100. Cynaeda dentalis (Denis \& Schiffermüller, 1775)

101. Cynaeda gigantea (Wocke, 1871)

102. Epascestria pustulalis (Hübner, 1823)

103. Tegostoma comparalis (Hübner, 1796) 
104. Titanio normalis (Hübner, 1796)

105. Achyra nudalis (Hübner, 1796)

106. Anania coronata (Leraut, 2005)

107. Anania crocealis (Hübner, 1796)

108. Anania funebris (Ström, 1768)

109. Anania fuscalis (Denis \& Schiffermüller, 1775)

110. Anania hortulata (Linnaeus, 1758)

111. Anania lancealis (Denis \& Schiffermüller, 1775)

112. Anania luctualis (Hübner, 1793)

113. Anania perlucidalis (Hübner, 1809)

114. Anania stachydalis (Germar, 1821)

115. Anania terrealis (Tretischke, 1829)

116. Anania testacealis (Zeller, 1847)

117. Anania verbascalis (Denis \& Schiffermüller, 1775)

118. Ecpyrrhorrhoe diffusalis (Guenée, 1854)

119. Ecpyrrhorrhoe rubiginalis (Hübner, 1796)

120. Euclasta splendidalis (Herrich-Schäffer, 1848)

121. Loxostege aeruginalis (Hübner, 1796)

122. Loxostege clathralis (Hübner, 1813)

123. Loxostege comptalis (Freyer, 1848)

124. Loxostege deliblatica (Szent-Ivány \& Uhrik-Meszáros, 1942)

125. Loxostege fascialis (Hübner, 1796)

126. Loxostege mucosalis (Herrich-Schäffer, 1848)

127. Loxostege sticticalis (Linnaeus, 1761)

128. Loxostege turbidalis (Treitschke, 1829)

129. Loxostege virescalis (Guenée, 1854)

130. Nascia cilialis (Hübner, 1796)

131. Ostrinia nubilalis (Hübner, 1796)

132. Ostrinia palustralis (Hübner, 1796)

133. Ostrinia quadripunctalis (Denis \& Schiffermüller, 1775)

134. Paracorsia repandalis (Denis \& Schiffermüller, 1775)

135. Paratalanta hyalinalis (Hübner, 1796)

136. Paratalanta pandalis (Hübner, 1825)

137. Psammotis pulveralis (Hübner, 1796)

138. Pyrausta aerealis (Hübner, 1793)

139. Pyrausta aurata (Scopoli, 1763)

140. Pyrausta castalis (Treitschke, 1829)

141. Pyrausta cingulata (Linnaeus, 1758)

142. Pyrausta coracinalis (Leraut, 1982)

143. Pyrausta despicata (Scopoli, 1763)

144. Pyrausta falcatalis (Guenée, 1854)

145. Pyrausta nigrata (Scopoli, 1763) 
146. Pyrausta obfuscata (Scopoli, 1763)

147. Pyrausta ostrinalis (Hübner, 1796)

148. Pyrausta purpuralis (Linnaeus, 1758)

149. Pyrausta sanguinalis (Linnaeus, 1767)

150. Pyrausta virginalis (Duponchel, 1832)

151. Sclerocona acutella (Eversmann, 1842)

152. Sitochroa palealis (Denis \& Schiffermüller, 1775)

153. Sitochroa verticalis (Linnaeus, 1758)

154. Uresiphita gilvata (Fabricius, 1794)

155. Donacaula forficella (Thunberg, 1794)

156. Donacaula mucronella (Denis \& Schiffermüller, 1775)

157. Schoenobius gigantella (Denis \& Schiffermüller, 1775)

158. Scirpophaga praelata (Scopoli, 1763)

159. Anarpia incertalis (Duponchel, 1832)

160. Cholius luteolaris (Scopoli, 1772)

161. Eudonia angustea (Curtis, 1827)

162. Eudonia delunella (Stainton, 1849)

163. Eudonia lacustrata (Panzer, 1804)

164. Eudonia laetella (Zeller, 1846)

165. Eudonia mercurella (Linnaeus, 1758)

166. Eudonia pallida (Curtis, 1827)

167. Eudonia phaeoleuca (Zeller, 1846)

168. Eudonia truncicolella (Stainton, 1849)

169. Eudonia vallesialis (Duponchel, 1832)

170. Gesneria centuriella (Denis \& Schiffermüller, 1775)

171. Heliothela wulfeniana (Scopoli, 1763)

172. Scoparia ambigualis (Treitschke, 1829)

173. Scoparia basistrigalis (Knaggs, 1866)

174. Scoparia ingratella (Zeller, 1846)

175. Scoparia italica (Turati, 1919)

176. Scoparia manifestella (Herrich-Schäffer, 1848)

177. Scoparia perplexella (Zeller, 1839)

178. Scoparia pyralella (Denis \& Schiffermüller, 1775)

179. Scoparia staudingeralis (Mabille, 1869)

180. Scoparia subfusca (Haworth, 1811]

181. Agrotera nemoralis (Scopoli, 1763)

182. Antigastra catalaunalis (Duponchel, 1833)

183. Cydalima perspectalis (Walker, 1859)

184. Diasemia reticularis (Linnaeus, 1761)

185. Diasemiopsis ramburialis (Duponchel, 1834)

186. Dolicharthria bruguieralis (Duponchel, 1833)

187. Dolicharthria punctalis (Denis \& Schiffermüller, 1775) 
188. Dolicharthria stigmosalis (Herrich-Schäffer, 1848)

189. Duponchelia fovealis (Zeller, 1847)

190. Herpetogramma licarsisalis (Walker, 1859)

191. Hodebertia testalis (Fabricius, 1794)

192. Mecyna asinalis (Hübner, 1819)

193. Mecyna balcanica (Slamka \& Plant, 2016)

194. Mecyna flavalis (Denis \& Schiffermüller, 1775)

195. Mecyna lutealis (Duponchel, 1833)

196. Mecyna trinalis (Denis \& Schiffermüller, 1775)

197. Metasia carnealis (Treitschke, 1829)

198. Metasia corsicalis (Duponchel, 1833)

199. Metasia ophialis (Treitschke, 1829)

200. Metasia rosealis (Ragonot, 1895)

201. Metasia suppandalis (Hübner, 1823)

202. Nomophila noctuella (Denis \& Schiffermüller, 1775)

203. Palpita vitrealis (Rossi, 1794)

204. Patania balteata (Fabricius, 1798) (=crocealis Duponchel, 1834)

205. Patania ruralis (Scopoli, 1763)

206. Spoladea recurvalis (Fabricius, 1775)

207. Udea accolalis (Zeller, 1867)

208. Udea alpinalis (Denis \& Schiffermüller, 1775)

209. Udea cyanalis (La Harpe, 1855)

210. Udea ferrugalis (Hübner, 1796)

211. Udea fimbriatralis (Duponchel, 1834)

212. Udea fulvalis (Hübner, 1809)

213. Udea languidalis (Eversmann, 1842)

214. Udea lutealis (Hübner, 1809)

215. Udea nebulalis (Hübner, 1796)

216. Udea numeralis (Hübner, 1796)

217. Udea olivalis (Denis \& Schiffermüller, 1775)

218. Udea prunalis (Denis \& Schiffermüller, 1775)

\section{Family Pyralidae}

219. Achroia grisella (Fabricius, 1794)

220. Galleria mellonella (Linnaeus, 1758)

221. Aphomia sociella (Linnaeus, 1758)

222. [Aphomia unicolor (Staudinger, 1880)]

223. Aphomia zelleri (Joannis, 1932)

224. Corcyra cephalonica (Stainton, 1866)

225. Lamoria anella (Denis \& Schiffermüller, 1775)

226. Anerastia lotella (Hübner, 1813)

227. Ematheudes punctella (Treitschke, 1833) 
228. Epidauria strigosa (Staudinger, 1879)

229. Epidauria transversariella (Zeller, 1848)

230. Hypsotropa limbella (Zeller, 1848)

231. Hypsotropa vulneratella (Zeller, 1847)

232. Peoria pectinella (Chrétien, 1911)

233. Valdovecaria umbratella (Treitschke, 1832)

234. Cryptoblabes bistriga (Haworth, 1811)

235. Cryptoblabes gnidiella (Millière, 1867)

236. Acrobasis advenella (Zincken, 1818)

237. Acrobasis bithynella (Zeller, 1848)

238. Acrobasis centunculella (Mann, 1859)

239. Acrobasis consociella (Hübner, 1813)

240. Acrobasis dulcella (Zeller, 1848)

241. Acrobasis fallouella (Ragonot, 1871)

242. Acrobasis glaucella (Staudinger, 1859)

243. Acrobasis getuliella (Zerny, 1914)

244. Acrobasis legatea (Haworth, 1811)

245. Acrobasis marmorea (Haworth, 1811)

246. Acrobasis obliqua (Zeller, 1847)

247. Acrobasis obtusella (Hübner, 1769)

248. Acrobasis porphyrella (Duponchel, 1836)

249. Acrobasis repandana (Fabricius, 1798)

250. Acrobasis romanella (Millière, 1870)

251. Acrobasis sodalella (Zeller, 1848)

252. Acrobasis suavella (Zincken, 1818)

253. Acrobasis tumidana (Denis \& Schiffermüller, 1775)

254. Alophia combustella (Herrich-Schäffer, 1855)

255. Amphithrix sublineatella (Staudinger, 1859)

256. Ancylosis cinnamomella (Duponchel, 1836)

257. Ancylosis oblitella (Zeller, 1848)

258. Ancylosis roscidella (Eversmann, 1844)

259. Apomyelois ceratoniae (Zeller, 1839)

260. Asalebria florella (Mann, 1862)

261. Assara conicolella (Constant, 1884)

262. Assara terebrella (Zincken, 1818)

263. Bradyrrhoa cantenerella (Duponchel, 1837)

264. Bradyrrhoa confiniella (Zeller, 1848)

265. Bradyrrhoa gilveolella (Treitschke, 1832)

266. Bradyrrhoa trapezella (Duponchel, 1836)

267. Cadra abstersella (Zeller, 1847)

268. Cadra calidella (Guenée, 1845)

269. Cadra cautella (Walker, 1863) 
270. Cadra figulilella (Gregson, 1871)

271. Cadra furcatella (Herrich-Schäffer, 1849)

272. Catastia marginea (Denis \& Schiffermüller, 1775)

273. Delplanqueia dilutella (Denis \& Schiffermüller, 1775)

274. Dectocera pseudolimbella (Ragonot, 1887)

275. Denticera divisella (Duponochel, 1842)

276. Dioryctria abietella (Denis \& Schiffermüller, 1775)

277. Dioryctria mendacella (Staudinger, 1859)

278. Dioryctria pineae (Staudinger, 1859)

279. Dioryctria robiniella (Millière, 1865)

280. Dioryctria schuetzeella (Fuchs, 1899)

281. Dioryctria simplicella (Heinemann, 1863)

282. Dioryctria sylvestrella (Ratzeburg, 1840)

283. Eccopisa effractella (Zeller, 1848)

284. Elegia atrifasciella (Ragonot, 1887)

285. Elegia fallax (Staudinger, 1881)

286. Elegia similella (Zincken, 1818)

287. Ephestia elutella (Hübner, 1796)

288. Ephestia kuehniella (Zeller, 1879)

289. Ephestia unicolorella subsp. woodiella (Richards \& Thomson, 1932)

290. Ephestia welseriella (Zeller, 1848)

291. Epischnia cretaciella (Mann, 1869)

292. Epischnia illotella (Zeller, 1839)

293. Epischnia leucoloma (Herrich-Schäffer, 1849)

294. Epischnia prodromella (Hübner, 1799)

295. Episcythrastis tabidella (Mann, 1864)

296. Episcythrastis tetricella (Denis \& Schiffermüller, 1775)

297. Etiella zinckenella (Treitschke, 1832)

298. Eucarphia vinetella (Fabricius, 1787)

299. Eurhodope cirrigerella (Zincken, 1818)

300. Eurhodope incompta (Zeller, 1847)

301. Eurhodope rosella (Scopoli, 1763)

302. Euzophera bigella (Zeller, 1848)

303. Euzophera cinerosella (Zeller, 1839)

304. Euzophera fuliginosella (Heinemann, 1865)

305. Euzophera osseatella (Treitschke, 1832)

306. Euzophera pinguis (Haworth, 1811)

307. Euzophera pulchella (Ragonot, 1887)

308. Euzopherodes charlottae (Rebel, 1914)

309. Euzopherodes lutisignella (Mann, 1869)

310. Euzopherodes vapidella (Mann, 1857)

311. Faveria dionysia (Zeller, 1846) 
312. Glyptoteles leucacrinella (Zeller, 1848)

313. Gymnancyla canella (Denis \& Schiffermüller, 1775)

314. Gymnancyla hornigii (Lederer, 1852)

315. Homoeosoma nebulella (Denis \& Schiffermüller, 1775)

316. Homoeosoma nimbella (Duponchel, 1837)

317. Homoeosoma sinuella (Fabricius, 1794)

318. Hypochalcia ahenella (Denis \& Schiffermüller, 1775)

319. Hypochalcia decorella (Hübner, 1810)

320. Hypochalcia dignella (Hübner, 1796)

321. Hypochalcia lignella (Hübner, 1796)

322. Insalebria serraticornella (Zeller, 1839)

323. Isauria dilucidella (Duponchel, 1836)

324. Khorassania compositella (Treitschke, 1835)

325. Matilella fusca (Haworth, 1811)

326. Merulempista amoenella (Zeller, 1848)

327. Merulempista brucella (Staudinger, 1879)

328. Merulempista cingillella (Zeller, 1846)

329. Metallosticha argyrogrammos (Zeller, 1847)

330. Metallostichodesbicolorella (Heinemann, 1864)

331. Metallostichodes nigrocyanella (Constant, 1865)

332. Moitrelia obductella (Zeller, 1839)

333. Myelois circumvoluta (Fourcroy, 1785)

334. Nephopterix angustella (Hübner, 1796)

335. Nyctegretis lineana (Scopoli, 1786)

336. Oncocera semirubella (Scopoli, 1763)

337. Ortholepis betulae (Goeze, 1778)

338. Oxybia transversella (Duponchel, 1836)

339. Pempelia albariella (Zeller, 1839)

340. Pempelia amoenella (Zeller, 1848)

341. Pempelia brephiella (Staudinger, 1879)

342. Pempelia compositella (Treitschke, 1835)

343. Pempelia palumbella (Denis \& Schiffermüller, 1775)

344. Pempeliella ornatella (Denis \& Schiffermüller, 1775)

345. Pempeliella sororiella (Zeller, 1839)

346. Phycita acericola (Kuznetzov, 1960)

347. Phycita coronatella (Guenée, 1845)

348. Phycita cryptica (Plant \& Slamka, 2016)

349. Phycita meliella (Mann, 1864)

350. [Phycita nephodeella (Ragonot, 1887)]

351. Phycita poteriella (Zeller, 1846)

352. Phycita roborella (Denis \& Schiffermüller, 1775)

353. Phycita torrenti (Agenjo, 1962) 
354. Phycitodes albatella (Ragonot, 1887)

355. Phycitodes bentickella (Pierce, 1937)

356. Phycitodes binaevella (Hübner, 1813)

357. Phycitodes inquinatella (Ragonot, 1887)

358. Phycitodes maritima (Tengström, 1848)

359. Plodia interpunctella (Hübner, 1813)

360. Psorosa dahliella (Treitschke, 1832)

361. Psorosa mediterranella (Amsel, 1954)

362. Psorosa nucleolella (Möschler, 1866)

363. Psorosa tergestella (Ragonot, 1901)

364. Pterothrixidia rufella (Duponchel, 1836)

365. Rhodophaea formosa (Haworth, 1811)

366. Sciota adelphella (Fischer von Röslerstamm, 1836)

367. Sciota fumella (Eversmann, 1844)

368. Sciota insignella (Mann, 1862)

369. Sciota rhenella (Zincken, 1818)

370. Selagia argyrella (Denis \& Schiffermüller, 1775)

371. Selagia spadicella (Hübner, 1796)

372. Selagia subochrella (Herrich-Schäffer, 1849)

373. Trachonitis cristella (Denis \& Schiffermüller, 1775)

374. Vitula biviella (Zeller, 1848)

375. Zophodia grossulariella (Hübner, 1809)

376. Endotricha flammealis (Denis \& Schiffermüller, 1775)

377. Hypotia corticalis (Denis \& Schiffermüller, 1775)

378. Hypotia massilialis (Duponchel, 1832)

379. Aglossa caprealis (Hübner, 1809)

380. Aglossa pinguinalis (Linnaeus, 1758)

381. Bostra obsoletalis (Mann, 1884)

382. Hypsopygia costalis (Fabricius, 1775)

383. Hypsopygia fulvocilialis (Duponchel, 1834)

384. Hypsopygia glaucinalis (Linnaeus, 1758)

385. Hypsopygia incarnatalis (Zeller, 1847)

386. Hypsopygia rubidalis (Denis \& Schiffermüller, 1775)

387. Loryma egregialis (Herrich-Schäffer, 1838)

388. Pyralis farinalis (Linnaeus, 1758)

389. Pyralis regalis (Denis \& Schiffermüller, 1775)

390. Stemmatophora brunnealis (Treitschke, 1829)

391. Stemmatophora combustalis (Fischer von Röslerstamm, 1842)

392. Stemmatophora honestalis (Treitschke, 1829)

393. Synaphe antennalis (Fabricius, 1794)

394. Synaphe bombycalis (Denis \& Schiffermüller, 1775)

395. Synaphe moldavica (Esper, 1794)

396. Synaphe punctalis (Fabricius, 1775) 